\title{
PSICOLOGÍA EDUCACIONAL, PSICOPEDAGOGÍA Y EDUCACIÓN ESPECIAL
}

\author{
LUIS BRAVO V. ${ }^{1}$ \\ Universidad Católica de Chile \\ (RECIBIDO EL 3/11/2009, ACEPTADO EL 2/12/ 2009)
}

\begin{abstract}
RESUMEN
El objetivo de este artículo es analizar las relaciones de la Psicología Educacional con la Psicopedagogía y con la Educación Especial a fin de aclarar mejor los roles de los respectivos profesionales frente a las dificultades del aprendizaje escolar.
\end{abstract}

Palabras clave: Psicología educacional, psicopedagogía, dificultades del aprendizaje, procesos cognitivos.

\section{ABSTRACT \\ EDUCATIONAL PSYCHOLOGY, PSICOPEDAGOGIA AND SPECIAL EDUCATION}

The aim of this article is to analise the relationships among the Psychological Education and the Psychopedagogy with the Special Education for learning disabled children and the work of the respective professionals.

Keywords: Psychological Education, Psychopedagogy, Learning disabilities, Cognitive processing.

\section{INTRODUCCIÓN}

Este objetivo se explica por el fuerte desarrollo que ha tenido en algunos países de habla castellana el ejercicio profesional de los psicopedagogos, formados en su mayoría, en las facultades de educación, a diferencia de los psicólogos educacionales, que se forman en las escuelas de psicología. Con frecuencia, el ejercicio profesional de ambos se ejerce de manera paralela y a veces ignorándose mutuamente, lo cual invita a realizar un análisis de los respectivos campos de acción científica y profesional.

Quiero partir diciendo que es difícil establecer límites precisos entre la Psicología Educacional, la Psicopedagogía y la Educación Especial. Las tres pueden describirse como "disciplinas en búsqueda de identidad", descripción que hizo Mena de la Psicología Educacional. Tanto una como la otra están en un proceso de "identidad en construcción" (Mena, 1991). Una dificultad para elaborar una delimitación de sus respectivas identidades

1 Profesor Honorario de la Universidad Mayor de San Marcos y Profesor Emérito de la Universidad Católica de Chile. E-mail: abravov@uc.cl 
reside en que recurren a bases teóricas comunes y tienen campos de aplicación convergente, lo cual produce a que entre ellas se origine una identidad parcialmente compartida, con límites imprecisos.

\section{La psicología educacional}

Esta construcción de la identidad de la Psicología Educacional, se deriva de diferencias históricas. La psicología educacional emergió con el siglo XX de manera paralela al desarrollo de la psicología, como ciencia autónoma, derivada de la psicología general, y su objetivo fue aportar a la educación los avances que aparecían en el campo de la experimentación de los procesos del aprendizaje, los conocimientos sobre el desarrollo infantil y los estudios diferenciales y posteriormente sobre los procesos cognitivos involucrados en el aprendizaje escolar.

A comienzos del siglo XX era una disciplina que intentaba aportar conocimientos y estrategias a la educación sin participar del currículum educativo ni de la formación de los maestros. Este aporte tenía por objetivo exigir a la pedagogía que adoptara bases más científicas para su quehacer educacional. Ausubel considera que es una disciplina aplicada a los problemas educativos, con una elaboración diferente de la psicología general, pues tiene un nivel menor de generalidad (Ausubel,1969, en Coll,1991).

Al decir de Coll, el éxito que tuvo la psicología educacional en la tarea de aportar un modelo científico de aproximación a los procesos educacionales, fue origen de que cincuenta años más tarde llegara "a ocuparse progresivamente de todos los problemas y aspectos relevantes el fenómeno educativo", lo cual hizo que sus contenidos se extendieran enormemente y sus límites se desdibujaran, perdiendo parcialmente su identidad inicial (Coll, 1991: 17). La búsqueda se esta identidad perdida habría motivado, posteriormente, a la psicología educacional, a adoptar enfoques interdisciplinarios, asumiendo como objeto de su estudio algunos aspectos propios del currículum escolar, tales como los programas de enseñanza, la influencia del medio escolar, o las características organizacionales del sistema educacional y la formación de los maestros.

El interés común por estos temas permitió establecer puentes entre la psicología educacional y la pedagogía, con objetivos compartidos por ambas. Sin embargo, el intento de lograr una "aprehensión global de fenómeno educativo" (Coll, 1991: 20), habría tenido como consecuencia para la psicología educacional, cierto abandono del tema del sujeto -de la psicología individual cognitiva y emocional del niño y del adolescente, en proceso de educarse- en beneficio del estudio del sistema escolar que lo cobija. Este abandono parcial de los sujetos -el niño y el adolescente- como objetivo primario, al ser reemplazado por un enfoque sistémico, habría dejado en algunos países espacio para el desarrollo de la "psicopedagogía", como disciplina psicológica y pedagógica mixta, aplicada a situaciones individuales.

\section{Psicología educacional y psicopedagogía}

En algunas partes ambas disciplinas han tenido un desarrollo paralelo, a veces ignorándose mutuamente. La psicología educacional se ha desarrollado principalmente en las escuelas 
de psicología, se ha planteado como objetivo principal enriquecer el quehacer pedagógico con investigaciones y nuevos aportes científicos derivados de metodologías propias. Un ejemplo de esta afirmación fue que años atrás un tema principal que abordaba la psicología Educacional eran los descubrimientos de Piaget. De ellos derivó una epistemología que explicaba la génesis del conocimiento y daba pautas a la educación sobre el desarrollo cognitivo normal de los niños. Posteriormente, el conocimiento del trabajo y de la publicaciones de Vigotsky modificó ese concepto de desarrollo cognitivo espontáneo, asociándolo al de interacción entre el niño y el medio, lo cual motivó a la psicología Educacional para que se interesara por el medio educacional y luego por el sistema escolar. La psicología psicoanalítica, por su parte, mostró la importancia del desarrollo emocional temprano y de las figuras parentales de identificación. De ahí que la psicología Educacional se interesara por la familia como factor determinante del desarrollo afectivo. Otro ejemplo fue la influencia de las corrientes conductistas, cuyos aportes se aplicaron a las metodologías de enseñanza como un proceso unilateral, que iba desde el profesor hacia el niño. Posteriormente, los aportes sistémicos han modificado esta orientación unilateral abordando el proceso de interacción entre aprendizaje activo de los niños y su medio pedagógico. Actualmente el mayor aporte de la psicología es la investigación de los procesos cognitivos, tales como el lenguaje, la memoria, la conciencia fonológica, que han permitido descubrir los aportes que tienen al aprendizaje del lenguaje escrito y a las dificultades para aprenderlo (Bravo, 2009).

La psicopedagogía, por su parte, formó parte desde el comienzo del currículum que imparten las escuelas de educación y pedagogía para el trabajo con niños que tienen dificultades para aprender. En los cursos de psicopedagogía se seguían preferentemente las corrientes europeas -francesas, belgas, suizas, alemanas y españolas- de la psicología pedagógica y su desarrollo tuvo un origen algo diferente que la psicología educacional. Partió del fenómeno educativo mismo de sus necesidades en el aula, considerándose una disciplina aplicada que utiliza los aportes de la psicología para enriquecer el quehacer educacional. Para la psicopedagogía, tal como se imparte hasta hoy día en las escuelas pedagógicas, la educación es su objetivo central y su intercambio con ella, su fuente de sustentación, sea en el trabajo con los niños, sea en el trabajo en el aula, o con los maestros. De este modo, por ejemplo, un enfoque psicológico piagetano o vigotskiano son utilizados en la medida en que su aplicación derive de una necesidad escolar y sea una contribución importante para mejorar el aprendizaje.

\section{La psicopedagogía como quehacer profesional}

El término psicopedagogía deriva probablemente de "psicología pedagógica", concepto ya utilizado en la década de los años cincuenta por Claparède en Suiza.

El Diccionario de la Real Academia Española define la "psicopedagogía" como "rama de la psicología que se ocupa de los fenómenos de orden psicológico para llegar a una formulación más adecuada de los métodos didácticos y pedagógicos.”

El Diccionario de Educación Especial de Brito, (1983), define "psicopedagogía” como "la pedagogía que está fundamentada esencialmente en la psicología como ciencia. 
Utiliza esta pedagogía, recursos psicológicos para hacerla más efectiva”. En una segunda definición cita a H. Pièron que la describió como una "Pedagogía científicamente basada en la psicología del niño".

De estas definiciones y descripciones se desprende que el término psicopedagogía se refiere a una especialización psicológica y pedagógica, cuya acción concreta consiste, principalmente, en la aplicación de los conocimientos derivados de ambas ciencias a problemas concretos que surgen en el campo de la educación.

Coll (1991), por su parte, estima que el término psicopedagogía designa los aspectos aplicados y profesionales de la psicología de la educación, sin delimitar su esfera de acción.

En un sentido más restringido, Brito (1991) también menciona los términos "psicopedagogía terapéutica" o "correctiva", coincidiendo en este aspecto, con la función profesional que ejercen los profesores de educación especial que atienden a los niños con trastornos escolares del aprendizaje y de la conducta.

Desde otra perspectiva, la psicopedagogía ha sido definida como "la disciplina que estudia la naturaleza y los procesos del aprendizaje humano, formal y contextualizado, y sus alteraciones " (Careaga, s/f: 145). Esta definición destaca como primer objetivo el estudio del "aprendizaje formal y contextualizado" que se realiza en un medio social determinado y también "sus alteraciones", o deficiencias por déficits en los "procesos que sustentan el aprendizaje", sean cognitivos, afectivo sociales y/o del entorno educativo. Para este autor "el hecho psicopedagógico" tendría dos pilares fundamentales: La "Investigación evaluativa de la naturaleza y los procesos del aprendizaje y sus alteraciones y "la intervención en dicho proceso". (Careaga, s/f: 147). Estos “dos pilares fundamentales” relacionan directamente la psicopedagogía con la educación especial.

La psicopedagogía como un quehacer profesional empezó en la década de los años cincuenta con la creación de algunas clínicas psicopedagógicas destinadas a atender a escolares con diferentes déficits en su desarrollo. Como hemos visto, su actividad profesional no derivó de la psicología educacional, sino del trabajo escolar con niños que requerían ayuda psicopedagógica especializada para mejorar su aprendizaje. En este sentido, la psicopedagogía aparece desde su origen estrechamente ligada al proceso del aprendizaje escolar y a las dificultades que presentan algunos niños que tienen un desarrollo atípico para seguir la escolaridad común.

La preocupación por las dificultades del aprendizaje como quehacer psicopedagógico, tuvo su origen en la necesidad escolar y social -no clínica- derivada de la gran cantidad de niños que repetían curso o abandonaban tempranamente las escuelas por no aprender a leer o a calcular. De la necesidad de abordar este problema con un criterio pedagógico y no psicológico, surgió su quehacer profesional destinado a conocer las causas de este problema y luego darles soluciones adecuadas. Algunas de sus soluciones son individuales, como sucede con los niños disléxicos, que no pueden aprender con metodologías de lectura colectivas y otras tienen enfoque pedagógico curricular, como sucede con los niños de aprendizaje lento. En estos ejemplos, la intervención psicopedagógica está destinada a solucionar problemas educativos originados en los mismos niños y que por lo tanto perturban 
también el proceso educacional. En otros casos pueden partir de acciones psicopedagógicas destinadas a solucionar problemas colectivos en el aula o de la comunidad escolar o a mejorar la labor pedagógica de los profesores.

En todos estos casos los aportes de las ciencias psicológicas pueden ser más proximales o más distales, según sea la naturaleza del problema y la formación que reciben los psicopedagogos. También depende de la formación que hayan recibido los psicólogos educacionales en temas relacionados con las dificultades del aprendizaje.

Su relación con la educación especial la veremos a continuación.

\section{La psicopedagogía y la educación especial}

La creación de la psicopedagogía como quehacer pedagógico autónomo significó una innovación dentro del ambiente educacional, ya que no sólo implicó la creación de una nueva especialidad profesional en el campo de la pedagogía, sino que implicaba una dura crítica a la rigidez de las metodologías de enseñanza que no asumían la diversidad psicológica de los niños. La creación de estos especialistas fue junto con proposiciones concretas para flexibilizar el currículum imperante en pedagogía básica, que favoreciera la integración real de los niños que presentaban dificultades y la proposición de nuevas metodologías de evaluación de sus habilidades para aprender.

La educación especial fue definida como una rama de la educación general, que tiene por finalidad educar a niños cuyas características psicológicas o físicas les impide tener éxito en el aprendizaje, dentro del sistema educacional normal y que necesitan un apoyo individual e institucional.

Por la naturaleza de su quehacer, esta especialidad está ubicada en los límites de la educación normal con la psicología educacional y con la neuropsicología cognitiva. Con la educación normal, pues actúa donde ésta no logra a cumplir sus objetivos más importantes en el aprendizaje y desarrollo de los niños. Con la psicología educacional y la neuropsicología cognitiva, requiere de ellas marcos teóricos de referencia e investigaciones empíricas para dar mayor solidez a su quehacer profesional. Esta especialización educacional no desconoce la importancia del contexto educativo ni tampoco de la condiciones socioculturales y familiares de los niños ya que están presentes en las estrategias de atención a cada niño.

La educación especial así concebida tiene por objetivo ayudar a los niños que presentan alguna diversidad que les impide tener un eficiente rendimiento escolar. Son alumnos que no logran los niveles de aprendizaje esperados, debido primariamente a interferencias originadas por algunos déficits cognitivos o verbales de su desarrollo. Se caracteriza por asumir la "diversidad" de esos niños, dentro del mismo sistema escolar, tratando de lograr la plena integración escolar de ellos al aula común. La intervención psicopedagógica especial tiene por objetivo darles la ayuda complementaria que les permita superar la segregación invisible que los aisla en el interior de las salas de clases.

Algunas de nuestras investigaciones muestran que, con mucha frecuencia, los niños de aprendizaje lento o los disléxicos, son ignorados durante la clase por sus propios maestros, quienes omiten interrogarlos, ayudarlos o corregirle sus trabajos, pasando a constituir un 
grupo invisible, que no es tomado en cuenta, en la medida en que no perturben la clase. Es decir, que la integración que busca la psicopedagogía especial debe efectuarse en primer lugar con los propios alumnos que tienen dificultades para seguir la clase.

En el caso de los niños con trastornos más severos de aprendizaje no se trata solamente de ayudarlos a integrarse, sino de evitar que esta segregación invisible origine repitencias de cursos y retiros prematuros no voluntarios. En este sentido, la educación especial debe utilizar estrategias psicopedagógicas derivadas de la psicología para ayudarlos, tanto en un trabajo individual, como también familiar. En consecuencia,sus métodos de trabajo son esencialmente "psicopedagógicos", y sus estrategias dependen mucho de los aportes que reciban de la psicología cognitiva, de la psicolingüística y de la neuropsicología.

En algunos lugares, los psicopedagogos y los profesores especiales actúan también como consultores de los maestros dentro de las escuelas, lo cual plantea una similitud de funciones con los psicólogos educacionales.

\section{La evolución de los modelos psicopedagógicos y de la psicología cognitiva}

Así como ha habido una evolución en las estrategias educacionales en los últimos treinta años, también hemos vivido una evolución muy significativa en el enfoque psicopedagógico de las dificultades escolares del aprendizaje, principalmente como consecuencia de las investigaciones en psicología educacional y en neuropsicología cognitiva. (Scagliotti y Pinto, 1997).

Hasta la década de 1970 predominaba en psicología y en pedagogía la influencia de las escuelas conductistas, cuya aplicación al campo escolar privilegiaba el aprendizaje de conductas observables y medibles, junto con métodos de enseñanza repetitivos y de contenidos asociados con respuestas controlables, cuya persistencia en el tiempo producía un aprendizaje estable. En cierta manera, este modelo implicaba que el desarrollo de los procesos mentales superiores dependería de la estimulación de los procesos inferiores y que hay cierta correlación entre el desarrollo mental de los niños y su aprendizaje escolar.

El objetivo prioritario de la Psicología educacional en esa época, era un "aprendizaje extrínseco", entendiendo por tal el condicionamiento, la memorización y/o la habituación de respuestas y de conductas, sin tomar en debida consideración cómo emergen y se elaboran los procesos superiores del pensamiento. El concepto de enseñar, derivado de esta corriente, no buscaba directamente ayudar a desarrollar modos de pensar, crear o razonar, sino más bien de retener y repetir. No se hablaba todavía de "aprendizajes significativos".

Por otra parte, el modelo metodológico que predominaba en educación especial en la década de los años setenta, privilegiaba un enfoque perceptivo-motor. Los déficits perceptivo visuales y psicomotores eran considerados claves explicativas de las dificultades para aprender. Cruickshank (1975) expresaba que la percepción permite que los estímulos cognitivos penetren al sistema nervioso y provoquen respuestas aprendidas o condicionadas. Las dificultades de aprendizaje se originarían en una dificultad para responder selectivamente a los estímulos, y el modelo de rehabilitación consistía en enseñar a los niños a ordenar y a controlar los estímulos que reciben. Esta posición era compartida, en cierta manera, 
por Frostig y Bender, cuyos trabajos y tests constituyeron un elemento importante en la formación de los especialistas de hace veinte años atrás. Los modelos de rehabilitación de la lectura, para los niños disléxicos, consistían principalmente en ejercicios asociativos entre percepciones visuales de las letras, sílabas o palabras, con su pronunciación, sin avanzar hacia los procesos psicolingüísticos subyacentes que dan significado a las palabras decodificadas. Los niños con dificultades en la lectura eran entrenados en reconocer y repetir incansablemente asociaciones visuales de los signos gráficos o de figuras geométricas, con su orientación espacial, lo que se suponía que les permitiría aprender a leer al saber discriminar sus diferencias espaciales o gráficas.

Esta situación histórica marcó fuertemente los marcos teóricos del trabajo profesional inicial de los psicopedagogos.

En la década siguiente se produjo un cambio fundamental, como consecuencia de los avances en psicología cognitiva y del énfasis en los procesos psicolingüísticos para enfocar las dificultades para aprender. Lohman (1989) expresó que: "La corriente predominante de la psicología se movió -suavemente- desde el condicionamiento a la percepción, y luego al pensamiento y a la resolución de problemas", lo que incidió en la elaboración de nuevos modelos psicopedagógicos y surgió la necesidad de desarrollar estructuras mentales más flexibles y creativas, que se reflejen en mejores logros en el aprendizaje en los niños con dificultades.

Otro avance conceptual de la psicología cognitiva con aplicación a la práctica psicopedagógica, ha sido la distinción entre los procesos y representaciones cognitivas superiores, y los procesos mediadores. Estos últimos son los que facilitan o permiten el acceso a los primeros y constituyen un objetivo más inmediato para la labor mediadora del profesor. Así por ejemplo, la acción pedagógica sobre la memoria inmediata verbal o sobre el reconocimiento fonológico, facilita la decodificación del significado y contribuye a mejorar la comprensión lectora.

Dentro de esta nueva perspectiva, el trabajo psicopedagógico con los procesos del pensamiento favorece nuevas reorganizaciones cognitivas, que amplían la capacidad para pensar y permite aprender contenidos de mayor complejidad. Los procesos involucrados en este tipo de aprendizaje significativo permiten discriminar, codificar, organizar, retener y decidir acerca de los contenidos de los programas escolares, de tal manera que cuando son aplicados facilitan la elaboración de nuevos esquemas conceptuales y facilitan el aprendizaje a los niños con déficits (Bravo, 1998).

Cuando el psicopedagogo tiene claridad acerca de cuáles son las estructuras cognitivas intermediarias que requieren intervención, y también cuáles son las limitaciones de los niños con los que está trabajando, puede acompañarlos en la secuencia que va desde la recepción inicial de la información que les está enseñando hasta su asimilación a nuevas estructuras del pensamiento.

Desde una perspectiva psicológica educacional, tiene mayor importancia en la evaluación psicopedagógica, la identificación de los procesos que los niños realizan que la suma de las respuestas correctas, ya que ellos pueden mostrar dónde están los razonamientos erróneos que realizan y aportar claves para intervenir en su corrección. 


\section{La interacción entre los procesos cognitivos y el aprendizaje escolar}

Desde una perspectiva educacional, las dificultades del aprendizaje de muchos niños, pueden describirse como resultante de una interacción fracasada entre las metodologías pedagógicas de enseñanza con las estructuras neuropsicológicas cognitivas del desarrollo individual. Este fracaso en la interacción se produce por un desajuste entre los procesos mentales que deben emplearse para dominar los aprendizajes requeridos y algunas dishabilidades de esos niños. En otros términos, se configura una situación en la cual se rompe la clásica dualidad "enseñanza- aprendizaje", quedando, por un lado, los objetivos de la enseñanza sin ser cumplidos y por otro, un aprendizaje insuficiente o deficitario.

En consecuencia, la psicología educacional la psicopedagogía o educación especial tienen principalmente por objetivo que los alumnos que ya fracasaron en los programas escolares, con las metodologías comunes de enseñanza o que presenten riesgo de un fracaso futuro, superen sus dificultades mediante la aplicación de estrategias de aprendizaje significativo y de adaptación escolar.

\section{Las diferencias socioeconómicas y culturales en la labor psicopedagógica}

Una situación frecuente que ocurre en la práctica profesional de la psicopedagogía en nuestro medio latinoamericano, es que las dificultades del aprendizaje escolar sean consideradas principalmente como un trastorno de origen neuropsicológico, cuando afectan a los niños de clase media o alta y como un problema derivado de insuficiencias socioculturales y económicas, en los niños de sectores populares. En el primer caso, la tendencia es ayudarlos mediante clases especiales, previo un diagnóstico psicológico o médico, y en el segundo, la tendencia ha sido tratar de modificar los programas escolares y los criterios de evaluación para hacerlos más adecuados con la realidad cognitiva de ellos.

Este sesgo parece derivar más de una percepción sociológica equivocada, que de la realidad psicológica de los niños que no aprenden. Tanto en los niños de clase media como en los provientes de niveles populares, el fracaso severo para aprender es un efecto individual, que debe ser enfocado individualmente, como lo muestran las investigaciones de Vellutino, según sean los orígenes de cada uno de ellos.

El aprendizaje escolar es un proceso neuropsicológico cognitivo que se efectúa en un contexto sociocultural. Las deficiencias en la calidad educacional de algunas escuelas o la impreparación de algunos maestros para detectar esas dificultades, pueden tener un peso explicativo en esta diferenciacion, pero ellas no excluyen las variables del desarrollo cognitivo individual. En consecuencia, un enfoque psicopedagógico integrado de las dificultades de aprendizaje debiera tomar en consideración tanto las características socioculturales y escolares colectivas, como las anormalidades neuropsicológicas del desarrollo de los niños. Pretender centrar casi todo el esfuerzo pedagógico en el contexto educacional sin aplicar estrategias derivadas de la Psicología Educacional, de la neuropsicología cognitiva o de las investigaciones originales de los propios maestros atenta contra el éxito de los resultados sobre los trastornos del aprendizaje.

En este punto, el quehacer de los psicopedagogos y maestros de educación especial, converge con la labor de los psicólogos educacionales, tanto en la evaluación de los déficits 
de los niños en los procesos cognitivos y psicolingüísticos de su aprendizaje, como en los provenientes de su interacción con los maestros y con currículum escolar.

\section{REFERENCIAS BIBLIOGRÁFICAS}

1. Bravo, L. (2009). Psicología de las dificultades de aprendizaje. Editorial Universitaria. Santiago de Chile.

2. Bravo, L. (2005). Lenguaje escrito y dislexias. Edit. Universidad Católica. Santiago de Chile.

3. Brito, A. (1983). Diccionario de educación especial. Universidad de Tarapacá, Arica.

4. Careaga, R. (s/f). Tradición y cambio en la psicopedagogía. Santiago de Chile: Universidad Educares. Bravo y Allende Editores.

5. Coll C. (1991). Psicología y educación: Aproximación a los objetivos y contenidos de la psicología de la educación. En: Desarrollo psicológico y Educación. Madrid: Alianza Editorial.

6. Cruickshank, W. y Hallahan, D. (Eds.) (1975). Perceptual and learning disabilities. Syracuse Univ. Press.

7. Lohman, D.F. (1989). Human intelligence: An introduction to advances in theory and research. Review of Educational Research, 59: 333-373.

8. Mena, I. (1991). ¿Qué es psicología educacional? Apuntes no publicados.

9. Scagliotti J. y Pinto A. (Eds.) (1997). Dificultades del aprendizaje: Avances en psicopedagogía. P. Santiago: PUC. 